\title{
RESISTENCIA Y LIBERTAD: UNA LECTURA DE "LAS COSAS QUE PERDIMOS EN EL FUEGO” DE MARIANA ENRÍQUEZ DESDE LAS PERSPECTIVAS DE FOUCAULT Y DE BEAUVOIR
}

\author{
RESISTANCE AND FREEDOM: A READING OF "LAS COSAS QUE \\ PERDIMOS EN EL FUEGO” BY MARIANA ENRÍQUEZ FROM THE \\ PERSPECTIVES OF FOUCAULT AND DE BEAUVOIR
}

\author{
Laura A. Sánchez \\ Universidad de Oslo. Noruega. \\ laura.azalia.s@gmail.com
}

\begin{abstract}
Resumen: En este trabajo analizo el aspecto de la resistencia femenina presente en "Las cosas que perdimos en el fuego" de Mariana Enríquez desde las perspectivas de Michel Foucault y Simone de Beauvoir. El primer autor sostiene que, a menudo, las expresiones de rebeldía son en el fondo mecanismos oficialmente permitidos y controlados que reafirman la autoridad de la que pretenden emanciparse. Por su parte, De Beauvoir asegura que en un sistema patriarcal las oportunidades de liberación que se ofrecen a las mujeres son ilusorias debido a que surgen de la lógica masculina. Con base en estas propuestas, me propongo observar en qué medida las acciones de las mujeres de la narración se oponen al orden que las domina y las lleva a trascender su situación, además de establecer un paralelo entre lo que sucede en el texto literario y lo que sucede en el contexto génesis del relato, tomando en cuenta que los textos de Mariana Enríquez son, de acuerdo con ella misma, textos políticos.
\end{abstract}

Palabras clave: Mariana Enríquez, Las cosas que perdimos en el fuego, resistencia, libertad, Michel Foucault, Simone de Beauvoir, feminismo, violencia, Latinoamérica, Argentina, literatura latinoamericana, literatura argentina, literatura de horror.

\footnotetext{
Abstract: In this work I analyze the aspect of the female resistance that is present in "Las cosas que perdimos en el fuego" by Mariana Enríquez from the perspectives of Michel Foucault and Simone de Beauvoir. The first author states that, usually, rebellious expressions are in bottom allowed and controlled by an official order. Therefore, they would be actually reasserting the order they pretend to get emancipated from. On the other hand, De Beauvoir affirms that, in a patriarchal system, the liberating choices offered to women are delusional because they arise from a masculine logic. Based on these proposals, I intent to observe to what extent the actions of the women in this story are in opposition to the dominant system and help them to transcend
} 
their situation. Additionally, I draw a parallel between what happens in the literary text and what happens in the context that surrounds it, given that Mariana Enriquez' texts are, according to herself, political texts.

Keywords: Mariana Enríquez, Things we lost in fire, resistance, freedom, Michel Foucault, Simone de Beauvoir, feminism, violence, Latin America, Argentina, Latin American literature, Argentinian literature, horror literature.

Recibido: 13/09/2018. Aceptado: 15/12/2019.

\section{Introducción}

$\mathrm{M}$ ariana Enríquez, nacida en Buenos Aires en 1973, es una de las escritoras argentinas que ha ganado más visibilidad en los últimos años a raíz de la publicación de dos antologías de cuentos: Los peligros de fumar en la cama (2009) y Las cosas que perdimos en el fuego (2016). Quizás el aspecto más comentado en la narrativa de Enríquez es su adscripción a la literatura de horror. En sus historias, Mariana Enríquez presenta personajes y situaciones cotidianos que poco a poco se transforman en escenas inquietantes.

En "Las cosas que perdimos en el fuego", el elemento cotidiano que sirve como punto de partida es el estado de riesgo en el que viven las mujeres de América Latina. Considero que el análisis de este texto es relevante porque aborda una situación desastrosa no solo para las propias mujeres sino para toda la sociedad. En esta realidad, la agresión y la intolerancia están completamente normalizadas, a pesar de que los discursos oficiales se empeñan en asegurar que la condición de las mujeres es cada vez mejor. En este sentido, las ideas de Foucault y De Beauvoir pueden contribuir a hacer una lectura crítica de la situación planteada en el texto y de su referente en la realidad.

El aspecto de la resistencia femenina implícito en "Las cosas que perdimos en el fuego" ha sido abordado con anterioridad, aunque no exhaustivamente, ya que la obra de Enríquez es reciente y por lo tanto aún no existe una gran cantidad de trabajos enfocados en esta autora o en el relato que me ocupa.

En el artículo intitulado "Desafiando al patriarcado a través del fuego: empoderamiento de las mujeres en 'Las cosas que perdimos en el fuego' 
de Mariana Enríquez”, Vanessa Rodríguez de la Vega resalta aspectos del texto tales como la biopolítica de los cuerpos bellos, la objetivación de la mujer, los feminicidios y el empoderamiento de la mujer ante tales circunstancias. Para Rodríguez, el hecho de que las mujeres de la historia decidan quemarse a sí mismas en un signo de subversión y empoderamiento, además, apela al significado simbólico del fuego y sostiene que este elemento podría funcionar en la narración como purificador del daño ocasionado por el orden patriarcal (Rodríguez, 2018: 146). En definitiva, para Rodríguez, las acciones que se presentan en "Las cosas que perdimos en el fuego" tienen una significación positiva porque a pesar de su naturaleza brutal, estarían encaminadas a romper con un esquema de violencia y opresión. Adicionalmente, la autora sugiere que dadas las circunstancias existe en el texto la esperanza de un nuevo orden en el que el ideal de belleza femenina ya no esté al servicio de los hombres.

En lo que toca a la configuración de los relatos de Mariana Enríquez, en la reseña intitulada "Frente a todos nuestros medios: la única mujer rebelde es la que arde”, María Celeste Cabral (2016) habla de la exageración como recurso utilizado por Enríquez para dibujar universos horrorosos. $\mathrm{Al}$ respecto, abunda: "sus cuentos se preguntan qué sucede más allá del límite en cualquier situación (...) Las historias juegan con la tensión entre la normalidad y sus extremos en una literatura exagerada e irónica que provoca gracia y terror" (Cabral, 2016: 125). Efectivamente, en "Las cosas que perdimos en el fuego" la exageración juega un papel importante en la construcción de la historia. Además, brinda una pauta para leer el texto a la luz de los postulados de Foucault y De Beauvoir, como explicaré a continuación.

\section{Resistencia y libertad}

\subsection{El concepto de resistencia según Foucault}

En la obra Historia de la sexualidad I. La voluntad de saber, Michel Foucault habla sobre el control que el orden social ejerce sobre ciertos aspectos de la existencia humana como la sexualidad. La idea extendida es que la sexualidad es un asunto reprimido porque se la considera inmoral. No obs- 
tante, un análisis histórico del tratamiento de la sexualidad deja ver que, en realidad, el orden social está excesivamente interesado en fomentar el discurso en torno a dicho tema con el fin de observar, analizar, clasificar y regular toda conducta que se le relacione. (Foucault, 2007: 20, 32-33, 37-38). A través de esta dinámica, se determina qué es lo "normal" y qué es lo anómalo, de manera que las manifestaciones sexuales alejadas de lo reglamentario no suponen una resistencia al orden social, sino que se convierten en un terreno vigilado y controlado por el poder (Foucault, 2007: 29, 56-57).

Para Foucault, la idea de la represión no tiene que ver con una "energía rebelde a la que habría que dominar". No existe "el juego entre una energía, salvaje, natural y viviente, que sin cesar asciende desde lo bajo, y un orden de lo alto que busca obstaculizarla" (Foucault, 2007: 99), sino que ambos elementos dan forma y sustento a las relaciones de poder. No se puede hablar de una heterogeneidad poder-resistencia, sino de un entramado en el que se localizan diferentes puntos de resistencia distribuidos irregularmente en el tiempo y el espacio. Dichos puntos funcionan como elementos de contraste que permiten aprehender o reconocer la lógica en la que se mueven las relaciones de poder y son de naturaleza cambiante, es decir, que lo que en una época se concibe como contrario al orden, en otra podría asimilarse como norma (Foucault, 2007: 116-117).

En resumen, para el análisis que me propongo realizar, entiendo el concepto de resistencia como un elemento integrado a las relaciones de poder y no como su contraparte externa. Su función es visibilizar las dinámicas de las relaciones de poder y su carácter puede variar en relación con el espacio o el tiempo en el que existe. El mecanismo descrito por Foucault atañe principalmente a la sexualidad, pero puede aplicarse a otros ámbitos en los que se hagan presentes las relaciones de poder y por eso es útil para analizar el relato de "Las cosas que perdimos en el fuego" en el que las dinámicas del poder toman la forma del patriarcado ${ }^{1}$.

${ }^{1}$ Para los efectos de este trabajo, utilizo el término patriarcado en su acepción más básica, es decir, como un sistema social en el que existe una dominación masculina sobre las mujeres (Pilcher y Whelehan, 2004: 93). 


\subsection{Simone de Beauvoir y la noción de libertad}

En El segundo sexo Simone de Beauvoir hace un recuento de la condición femenina a través de la historia para mostrar cómo las mujeres han sido sistemáticamente relegadas al papel del "otro" con respecto de los hombres y por ende, no han tenido las mismas posibilidades que éstos de existir como individuos plenos. Hay que recordar que el pensamiento de De Beauvoir se inscribe en la filosofía existencialista y por tanto, para ella, la existencia humana implica "una necesidad indefinida de trascenderse a sí mismo y de involucrarse en proyectos libremente elegidos" (De Beauvoir, 1997: 29; traducción personal)².

El drama de la mujer, según De Beauvoir, "reside en este conflicto entre las aspiraciones fundamentales de cada sujeto (...) - que siempre se considera a sí como esencial-y las exigencias de una situación en la que ella no es esencial" (De Beauvoir, 1997: 29). Es decir, que existe una contraposición entre el impulso natural de la mujer, en tanto ser humano, de trascenderse a sí misma, y las funciones que la sociedad - diseñada en términos masculinos - le ha asignado, las cuales son limitantes.

En este tenor, De Beauvoir reflexiona sobre uno de los argumentos antifeministas de su época: "La situación de la mujer no ha impedido nunca el florecimiento de grandes personalidades femeninas" (De Beauvoir, 1997: 164; traducción personal) ${ }^{3}$. El fallo en este razonamiento es que el éxito de unas cuantas mujeres no puede justificar la situación general del resto de ellas, y, por otro lado, el hecho de que estas "grandes personalidades femeninas” sean tan pocas es en sí mismo una prueba de las dificultades adicionales que las mujeres tienen que sortear para llegar a la misma posición que un hombre. Por estas razones, dice De Beauvoir, las mujeres reclaman que "por fin, se les concedan los derechos abstractos y las posibilidades concretas sin cuya conjugación la libertad no es más que una falsedad" (De Beauvoir, 1997: 164; traducción personal)4.

\footnotetext{
${ }^{2}$ El texto citado dice: "an undefined need to transcend himself, to engage in freely chosen projects” (De Beauvoir, 1997: 29).

${ }^{3}$ El texto citado dice: "the situation of woman has never prevented the flowering of great feminine personalities" (De Beauvoir, 1997: 164).

${ }^{4} \mathrm{El}$ texto citado dice: "at last the abstract rights and concrete possibilities without the concurrence of which liberty is only a mockery” (De Beauvoir, 1997: 164).
} 
Lo que me interesa resaltar en este caso es la división implícita en las afirmaciones de De Beauvoir: si existen "posibilidades concretas", entonces también existen posibilidades inconcretas, y si la ausencia de aquellas implica una libertad que no es "más que una falsedad", entonces también existe una libertad "verdadera". Un ejemplo de esto es la condición de algunas mujeres de la época en la que De Beauvoir escribió El segundo sexo (1948-1949). Estas personas contaban con tres beneficios que sus antecesoras no habían conocido: el derecho al voto, la posibilidad de tener un trabajo remunerado y una ley que ya no contemplaba la obediencia como obligación de la esposa, no obstante, como observa De Beauvoir, esto no implicaba necesariamente una liberación:

No es de suponerse, sin embargo, que la mera combinación del derecho al voto y al trabajo constituye una emancipación completa: el trabajo hoy no es la libertad. La estructura social no ha sido modificada suficientemente por los cambios en la condición de la mujer: este mundo, perteneciente siempre a los hombres, aún conserva la forma que ellos le han dado (De Beauvoir, 1997: 689 traducción personal) ${ }^{5}$.

La libertad de estas mujeres podría considerarse una falsedad porque consistía en la posibilidad de elegir entre trabajar en casa sin remuneración, o trabajar en una fábrica recibiendo un mal salario y sin renunciar a sus actividades domésticas (De Beauvoir, 1997: 690). Es decir, que sus opciones no eran realmente emancipadoras. La definición puntual de la "libertad falsa" y la "libertad verdadera" en el contexto de las relaciones de género rebasa los objetivos de este trabajo, pero la dicotomía planteada por De Beauvoir es útil para observar hasta qué punto las acciones emprendidas por las mujeres del relato que me ocupa contribuyen a su liberación.

${ }^{5} \mathrm{El}$ texto citado dice: "It is not to be supposed, however, that the mere combination of the right to the vote and job constitutes a complete emancipation: working today is not liberty. The social structure has not been much modified by the changes in woman's condition: this world, always belonging to men, still retains the form they have given it." (De Beauvoir, 1997: 689) 


\section{Resistencia y libertad en "Las cosas que perdimos en el fuego"}

A diferencia de otras de las historias Enríquez, y a pesar de que ésta es conocida como una autora del género de horror, "Las cosas que perdimos en el fuego" no presenta un elemento sobrenatural que cree una atmósfera de miedo. Lo que sí aparece es la referencia a un suceso de la vida cotidiana que se explora, se exagera y se lleva a un límite en donde la razón ya no puede explicar satisfactoriamente lo que está sucediendo.

La trama de "Las cosas que perdimos en el fuego" es dada a conocer por un narrador heterodiegético que habla en tercera persona y presenta la perspectiva de Silvina, una joven angustiada por lo que sucede en su entorno. En el cuento se relata cómo los casos de unas mujeres que fueron quemadas intencionalmente por sus parejas masculinas en plan de represalia provocan que otras mujeres tomen la decisión de incendiarse a sí mismas. Entre más episodios de feminicidios o violencia en contra de las mujeres se dan a conocer, más mujeres optan por prenderse fuego. Aunque al principio los incidentes son aislados y se llevan a cabo en privado, con el tiempo las mujeres se organizan y adoptan el nombre de Mujeres Ardientes. Dentro de este colectivo surgen "las hogueras", que son quemas de mujeres arregladas con el apoyo de otras mujeres y tienen todas las características de un ritual de iniciación. Las quemas no tienen la intención de matar a quienes eligen pasar por el proceso, sino sólo marcarlas o deformarlas, así que también van surgiendo hospitales clandestinos a donde estas mujeres pueden ir a recuperarse de sus quemaduras.

En un primer momento nadie puede creer que las mujeres se estén quemando a sí mismas. Pasada la incredulidad, la situación se vuelve un asunto público: el Estado responde con controles policíacos y, en general, con una vigilancia excesiva, pero nada de esto detiene la ola de féminas quemadas ni amedrenta a quienes las ayudan en su cometido. Silvina se mueve entre dos mundos: por un lado, siente indignación por las mujeres que han sido violentadas por sus parejas; ayuda a su madre -quien dirige uno de los hospitales clandestinos- y hasta filma una de las ceremonias para darla a conocer públicamente con el fin de que el movimiento gane credibilidad. A pesar de todo esto, no se une de lleno a las Mujeres Ardientes: está llena de dudas y llega a pensar que el movimiento es una locura y que quizás sea mejor traicionarlo y acabar con él desde adentro. Casi una año después de 
haber comenzado con "las quemas", las primeras sobrevivientes comienzan a salir a la luz y a tratar de llevar una vida normal con sus nuevos cuerpos deformados. Cuando el relato termina, Silvina sigue llena de rabia y de confusión, mientras que su madre y la amiga de ésta fantasean con que las quemas continúen indefinidamente e, incluso, con que Silvina protagonice una de ellas.

A primera vista, Enríquez presenta un mundo de mujeres valientes, independientes y decididas que toman el control de su existencia y de sus cuerpos en un orden eminentemente masculino. Sin embargo, hay indicios que apuntan a que esto es sólo una ilusión. Podría decirse que, en realidad, lo que Mariana Enríquez presenta es un universo horroroso en el que es prácticamente imposible escapar de la influencia del patriarcado.

Si se analiza el texto desde la perspectiva foucaultiana, se observa que existe un entramado de relaciones de poder en el que el sexo masculino es favorecido. La primera mujer quemada que se nombra en la narración -la chica del subte - fue agredida por su marido (Juan Martín Pozzi), porque ella le era infiel y él quería evitar que "fuera" (Enríquez, 2017: 186) de alguien más. Mientras ella estaba en el hospital sin poder hablar, él declaró que ella misma se había quemado y nadie lo puso en duda, a pesar de lo absurdo de su explicación (Enríquez, 2017: 186). En este tipo de lógica y de acciones, se nota que la mujer es concebida como un objeto susceptible de poseerse, y que puede o merece ser castigada si no se ajusta a dicha concepción. Adicionalmente, cuando se busca un responsable por este tipo de agresiones, no se sospecha del hombre, así que por un lado se niega, aunque sea temporalmente, que los hombres puedan ejercer este tipo de violencia, y por el otro, se responsabiliza casi automáticamente a las propias mujeres por lo que les sucede, lo cual implica, incluso, la atribución de una especie de locura intrínsecamente femenina.

La segunda mujer quemada es Lucila, una hermosa modelo que disfrutaba de fama relativa hasta que comenzó una relación amorosa con Mario Ponte, un futbolista de renombre. A partir de ese momento, ella se vuelve más conocida y consigue mejores contratos de trabajo (Enríquez, 2017: 188). En la narración, esto podría indicar una ventaja del sexo masculino sobre el femenino, ya que Lucila obtiene visibilidad y un estatus más favorable sólo a través de su relación con Mario. En otras palabras, para alcanzar el éxito en el universo que presenta Mariana Enríquez, es necesa- 
rio contar con un intermediario o aliado masculino. Trágicamente, el final de Lucila también está determinado por las acciones masculinas, como si Mario fuera a la vez creador y destructor de esta mujer.

Las acciones de Mario Ponte sirven como ejemplo a otros hombres que perpetúan y amplían la situación de abuso y desigualdad en toda la Argentina, como describe el narrador: "Hombres quemaban a sus novias, esposas, amantes, por todo el país. Con alcohol la mayoría de las veces, como Ponte (por lo demás el héroe de muchos), pero también con ácido" (Enríquez, 2017: 189). La violencia descrita en este fragmento presenta un flujo muy claro. No es un problema generalizado que afecte a toda la sociedad por igual, sino que se trata específicamente de hombres agrediendo a sus parejas femeninas. En este orden de ideas, y siguiendo la lógica de Foucault, las Mujeres Ardientes funcionan como puntos de resistencia. Su motivación y la manera en la que actúan permite aprehender en qué dirección se mueven las redes de relaciones de poder, no obstante, ellas no contradicen dicho poder, sino que lo refuerzan, le ofrecen nuevos terrenos para actuar.

La respuesta del Estado ante las acciones de las Mujeres Ardientes es el ejercicio de un control exacerbado, por ejemplo, cuando Silvina se ofrece a filmar una de las quemas, evita comprar una cámara nueva porque teme que la rastreen, mientras que los hospitales clandestinos son objeto de allanamientos (Enríquez, 2017: 193-194). Esta situación se condensa en una de las observaciones del narrador: "las mujeres sin familia o que sencillamente andaban solas por la calle caían bajo sospecha: la policía les hacía abrir el bolso, la mochila, el baúl del auto cuando ellos lo deseaban, en cualquier momento, en cualquier lugar (...) Muchas mujeres trataban de no estar solas en público para no ser molestadas por la policía. Todo era distinto desde las hogueras" (Enríquez, 2017: 194-195). En esta cita hay varios elementos llamativos: en primer lugar, se observa la creación de una suerte de estereotipo de las Mujeres Ardientes: mujeres solas y sin familia. Con base en esta idea preconcebida, se juzga y se acosa a las féminas, además, la policía puede invadir su privacidad de manera arbitraria y por último, las mujeres buscan la compañía masculina para no ser molestadas por la policía, o sea que su tranquilidad estaría condicionada por la presencia masculina. En resumen, las hogueras no mejoran la situación de las mujeres de este relato, por el contrario, reducen aun más su libertad.

La resistencia presente en "Las cosas que perdimos en el fuego" es, tal 
y como describe Foucault, en La historia de la sexualidad, flexible y móvil. A pesar de que el relato abarca poco más de un año, es posible ver cómo en este lapso las Mujeres Ardientes, sobre todo sus líderes, comienzan a agruparse en torno a unas ideas cuyo origen se remonta al orden que tratan de combatir, o sea, el patriarcado. Como señala Simone de Beauvoir en $E l$ segundo sexo, un orden social de esta naturaleza implica ciertas expectativas, como por ejemplo, la belleza, que aunque puede variar en su concepto, siempre ha estado presente a lo largo de la historia y ha contribuido a la cosificación de la mujer (De Beauvoir, 1997: 189).

Un indicio de la presencia de esta expectativa de belleza enraizada en el patriarcado se presenta cuando la chica del subte es entrevistada durante una manifestación: "-Si siguen así, los hombres se van a tener que acostumbrar. La mayoría de las mujeres van a ser como yo, si no se mueren. Estaría bueno, ¿no? Una belleza nueva." (Enríquez, 2017: 190). Se nota un dejo de sarcasmo en estas declaraciones. Sin embargo, la idea de una nueva forma de belleza va tomando forma a lo largo del relato y se aprecia de manera concreta cuando la madre de Silvina y la amiga de ésta, María Helena, conversan entre ellas: "Silvina solamente escuchó que ellas estaban demasiado viejas, que no sobrevivirían a una quema (...) pero Silvinita, ah, cuándo se decidiría Silvinita, sería una quemada hermosa, una verdadera flor de fuego" (Enríquez, 2017: 197). Como puede verse, lo que comenzó como un supuesto acto de rebeldía en contra de un modelo dominante se transforma, a su vez, en un patrón de conducta que se concibe como deseable y espera ser reproducido. En otras palabras, crea nuevas expectativas, pero siempre en torno a un ideal de belleza.

"Las cosas que perdimos en el fuego" leído desde la perspectiva beauvoiriana, también revela que el activismo de las Mujeres Ardientes está lejos de contraponerse realmente al sistema patriarcal dominante. Como mencioné, para De Beauvoir (1997) la existencia humana sólo se da plenamente cuando el individuo se trasciende a sí mismo mediante su involucramiento en proyectos libremente elegidos. En el caso de las mujeres, esta posibilidad no está a su alcance porque su histórica posición de desventaja frente a los hombres les asigna funciones limitantes y por lo tanto contrarias a su esencia individual. Las oportunidades de liberación que se ponen al alcance de las mujeres de este relato no son reales y tampoco lo es la libertad que supuestamente alcanzan, como explico a continuación. 
En primer lugar, los actos de las Mujeres Ardientes son una reacción a la forma en la que son tratadas por los hombres. Las hogueras no son un proyecto que surja de manera libre o espontánea con el fin único de que las mujeres se trasciendan a sí mismas. Aun cuando las Mujeres Ardientes se muestran decididas en algunos aspectos, su resolución es, en términos beauvorianos, sintomática de una libertad falsa, y el discurso de María Helena expone esto con toda claridad: "-Las quemas las hacen los hombres, chiquita, siempre nos quemaron. Ahora nos quemamos nosotras. Pero no nos vamos a morir: vamos a mostrar nuestras cicatrices" (Enríquez, 2017: 192.) De acuerdo con este pensamiento, las mujeres del relato son "libres" de elegir entre dos opciones: ser quemadas por sus parejas y morir (o quedar deformes), o quemarse ellas mismas y vivir para mostrar con orgullo sus cicatrices. Dicho de otro modo, no tienen posibilidades auténticas frente a ellas. Por más contestatarias y radicales que parecen sus decisiones, éstas no las llevan fuera del sistema que en un principio las puso en una situación desfavorable, peor aún: las convierte en sus propias victimarias. En síntesis, la resistencia que ofrecen las mujeres de este relato no las hace libres, ni las acerca a la libertad como ellas creen.

\section{Las hogueras arden más allá de la ficción: el paralelo entre el universo ficticio de "Las cosas que perdimos en el fuego" y el contexto actual}

Además de los elementos de las filosofías foucaultiana y beauvoiriana que pueden encontrarse en la configuración de "Las cosas que perdimos en el fuego", es relevante comentar los paralelos que existen entre este relato y el contexto social en el que surge. Es claro que la violencia a la que reaccionan las Mujeres Ardientes se corresponde con la que sufren cotidianamente las mujeres de América Latina, asimismo, están presentes otros elementos relacionados con la influencia que ejerce el patriarcado de manera global, como por ejemplo, el ideal de belleza que las mujeres son presionadas por alcanzar. Como se ve, no existe una gran diferencia entre el dolor o la incomodidad a los que se someten las mujeres de nuestra sociedad para alcanzar dichos parámetros y el dolor al que se someten las mujeres de la narración para llegar a ser "verdaderas flores de fuego". 
Resulta interesante observar también que la solución al problema de la violencia contra las mujeres en el universo narrativo de Mariana Enríquez recae en las propias mujeres: son ellas las que deben organizarse y buscar la forma de contrarrestar la situación, mientras que el colectivo masculino no asume ninguna responsabilidad, no se espera nada de él y hasta continúa con sus hábitos, dado que las mujeres victimizadas por sus parejas siguen apareciendo. De manera similar, en el orden de cosas en el que vivimos, las estrategias de prevención de violencia masculina en contra de las mujeres van dirigidas a éstas, en vez de enfocarse en los sujetos que ejercen dicha violencia.

Es aquí, en el punto de encuentro entre la realidad y la ficción, donde se hace más evidente el horror de este relato. Quizá en "Las cosas que perdimos en el fuego" no existe un elemento sobrenatural como en otras narraciones de Mariana Enríquez, pero sí existe, como señala Celeste Cabral (2016), el elemento de la exageración, que nos hace preguntarnos qué sucedería si lleváramos nuestra realidad al extremo o, mejor dicho, en dónde se encuentran los extremos de nuestra realidad y qué tan lejos estamos de tocarlos. También cabría preguntarse qué tan libres son las mujeres de nuestra sociedad con respecto a las posibilidades que se ponen a su alcance y qué tan concretas son las mejoras en la condición femenina en comparación con otras épocas de la historia. La respuesta no parece ser alentadora, de hecho, entre más de cerca se mira la situación, más evidente resulta que la influencia del patriarcado es omnipresente y, aparentemente, infinita.

\section{Conclusiones}

Las nociones de resistencia y libertad tal como las conciben Michel Foucault y Simone de Beauvoir, respectivamente, se encuentran presentes en el relato de "Las cosas que perdimos en el fuego". Lo anterior significa que las acciones de las mujeres que aparecen en el texto son en la superficie un indicio de rebeldía en contra de un sistema que las oprime, pero en el fondo se trata de acciones que surgen de la misma lógica del poder en turno y que por lo tanto no lo combaten, antes bien, refuerzan su dominio. Tales acciones surgen como un contrapunto del sistema en vigor, pero con el tiempo evolucionan e imitan el papel controlador de este. Finalmente, lo 
que parece ser una decisión emancipadora tomada por estas mujeres las conduce a una libertad falsa, puesto que las limita a escoger entre opciones generadas por el mismo sistema dominante en vez de brindarles la posibilidad de crear alternativas basadas en sus propias necesidades y creencias.

El horror, presente en el relato a través del elemento de la exageración, da pie a una crítica de la realidad histórica en la cual surge "Las cosas que perdimos en el fuego", específicamente, permite cuestionar hasta qué punto las mujeres pueden ser libres en un sistema predominantemente patriarcal y si las conductas liberadoras que se les ofrecen como opción no son más que una extensión del yugo que se les impone.

\section{Referencias}

Cabral, C. (2016). Frente a todos nuestros medios: la única mujer rebelde es la que arde El toldo de Astier. Propuestas y estudios sobre enseñanza de la lengua y la literatura. Recuperado de: http://sedici.unlp.edu. ar/bitstream/handle/10915/57110/Documento_completo.pdf-PDFA. pdf?sequence $=1$ \&isAllowed $=\mathrm{y}$

De Beauvoir, S. (1997). The second sex, London: Vintage Classics.

Enríquez, Mariana (2017). Las cosas que perdimos en el fuego en Las cosas que perdimos en el fuego, Nueva York: Vintage Español.

Foucault, Michel (2007). Historia de la sexualidad 1. La voluntad de saber, México: Siglo XXI.

Pilcher, J. y Whelehan, I. (2004). 50 key concepts in Gender Studies, London: SAGE Publications.

Rodríguez de la Vega, Vanesa (2018). Desafiando al patriarcado a través del fuego: el empoderamiento de las mujeres en Las cosas que perdimos en el fuego de Mariana Enríquez. Transmodernity: Journal of Peripheral Cultural Production of the Luso-Hispanic World, 8 (1). Recuperado de: https://escholarship.org/uc/item/2mx6c3s1 